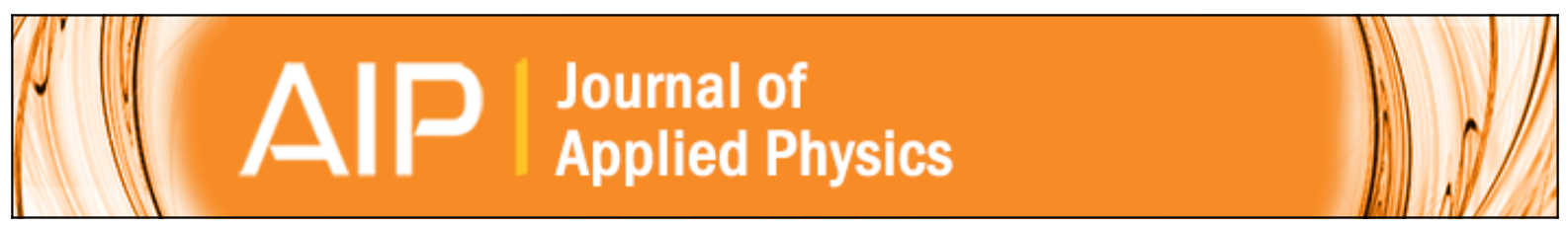

\title{
Parallel-plate waveguide with sinusoidally perturbed boundaries
}

Ali H. Nayfeh and Omar R. Asfar

Citation: Journal of Applied Physics 45, 4797 (1974); doi: 10.1063/1.1663138

View online: http://dx.doi.org/10.1063/1.1663138

View Table of Contents: http://scitation.aip.org/content/aip/journal/jap/45/11?ver=pdfcov

Published by the AIP Publishing

\section{Articles you may be interested in}

Multipactor saturation in parallel-plate waveguides

Phys. Plasmas 19, 072304 (2012); 10.1063/1.4736852

Study of the impedance mismatch at the output end of a $\mathrm{THz}$ parallel-plate waveguide

Appl. Phys. Lett. 100, 111120 (2012); 10.1063/1.3695329

Terahertz microfluidic sensor based on a parallel-plate waveguide resonant cavity

Appl. Phys. Lett. 95, 171113 (2009); 10.1063/1.3251079

$\mathrm{THz}$ transmission characteristics of dielectric-filled parallel-plate waveguides

J. Appl. Phys. 101, 083115 (2007); 10.1063/1.2719669

Radiation into anisotropic plasma from flanged parallel-plate waveguide

J. Appl. Phys. 45, 2493 (1974); 10.1063/1.1663620

\section{MIT LINCOLN}

\section{LABORATORY} CAREERS

\section{Discover the satisfaction of} innovation and service to the nation
- Space Control

- Air \& Missile Defense

- Communications Systems \& Cyber Security

- Intelligence, Surveillance and

Reconnaissance Systems

- Advanced
Electronics
- Tactical Systems
" Homeland
Protection
- Air Traffic Control

Massachusetts InStitute of TechNology

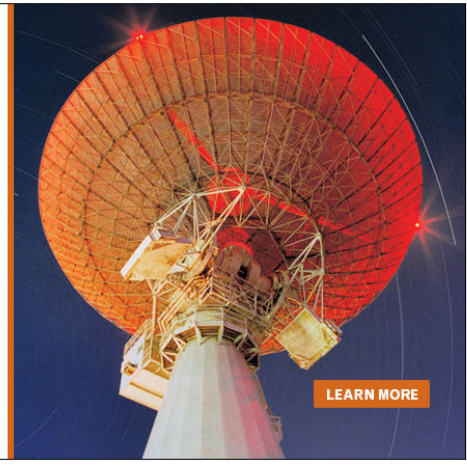




\title{
Parallel-plate waveguide with sinusoidally perturbed boundaries
}

\author{
Ali H. Nayfeh and Omar R. Asfar \\ Department of Engineering Science and Mechanics, Virginia Polytechnic Institute and State University, Blacksburg, \\ Virginia 24061 \\ (Received 30 May 1974) \\ The method of multiple scales is used to obtain a uniformly valid asymptotic expansion for the \\ propagation of TM modes on a parallel-plate waveguide with perfectly conducting boundary surfaces \\ that are sinusoidally perturbed in the direction of propagation. The analysis shows that resonance \\ occurs whenever the wave number of the wall distortion function is equal to the difference between \\ the wave numbers of two propagating modes. It is further shown that the generated mode is the \\ same order of magnitude as the excited mode due to resonance and that energy is continuously \\ exchanged between the two modes as they propagate down the guide.
}

\section{INTRODUCTION}

In this paper we investigate the generation of a propagating mode as a result of the interaction of an excited mode with the geometrical perturbations of the walls of a parallel-plate waveguide. The walls are assumed to be perfectly conducting and have wall distortion functions of the form

$$
\begin{aligned}
& \bar{x}=a \sin \left(\tilde{k}_{w} \tilde{z}\right) \quad \text { lower wall, } \\
& \tilde{x}=d+a \sin \left(\tilde{k}_{w} \tilde{z}+\theta\right) \quad \text { upper wall, }
\end{aligned}
$$

where $a$ is the amplitude of the wall's sinusoidal distortions, $d$ is the undistorted separation of the plates, $k_{w}$ is the wave number of the distortions, and $\theta$ is the phase difference between the distortions of the two walls. We treat the case of weak perturbations so that $a \ll d$.

The problem of a dielectric slab waveguide having wall distortion functions as those of Eqs. (1a) and (1b) was treated by Marcuse ${ }^{1}$ He considered the case of a slab with periodically varying thickness $(\theta=\pi)$, and used an ad hoc approximation in order to derive equations for the amplitudes of two interacting modes satisfying the resonance condition

$$
\tilde{k}_{m}=\tilde{k}_{n}-\tilde{k}_{w},
$$

where $\widetilde{k}_{m}\left(\tilde{k}_{n}\right)$ are the wave numbers of the generated (excited) mode. It is the purpose of the present analysis to treat the problem by systematic perturbation techniques taking into account the case of near resonance; that is

$$
\tilde{k}_{m} \approx \tilde{k}_{n}-\bar{k}_{w},
$$

and also for any value of $\theta$.

Following Nayfeh's treatment ${ }^{2}$ of acoustic waves in two-dimensional ducts with sinusoidal walls, we use the method of multiple scales ${ }^{3}$ to determine an approximate solution for the cases of TM and TE modes. To this end, we make length and time dimensionless using the average separation of the plates $d$ so that Eqs. (1a) and (1b) take the form

$$
\begin{aligned}
& x=\epsilon \sin \left(k_{w} z\right) \quad \text { lower wall, } \\
& x=1+\epsilon \sin \left(k_{w} z+\theta\right) \quad \text { upper wall, }
\end{aligned}
$$

where $x$ and $z$ are dimensionless coordinates and $\epsilon=a / d$ is a parameter much smaller than unity. We consider the case of TM modes as an illustration of the technique. The fields for a TM mode are derivable from a vector potential which for $H_{z}=0$ is $z$ directed. For a harmonic time variation $\exp (-i \omega t)$, the $z$-directed wave function $\psi$ is governed by the Helmholtz equation

$$
\nabla^{2} \psi+k^{2} \psi=0
$$

where $\nabla^{2}=\partial^{2} / \partial x^{2}+\partial^{2} / \partial z^{2}$ and $k$ is the free-space dimensionless wave number. The boundary conditions on $\psi$ for the case of infinite conductivity are the vanishing of the tangential component of the electric field at the boundaries. This gives

$$
\begin{gathered}
\left(\frac{\partial^{2}}{\partial z^{2}}+k^{2}\right) \psi=-\epsilon k_{w} \cos \left(k_{w} z\right) \frac{\partial^{2} \psi}{\partial x \partial z} \text { at } x=\epsilon \sin \left(k_{w} z\right), \\
\left(\frac{\partial^{2}}{\partial z^{2}}+k^{2}\right) \psi=-\epsilon k_{w} \cos \left(k_{w} z+\theta\right) \frac{\partial^{2} \psi}{\partial x \partial z} \\
\text { at } x=1+\epsilon \sin \left(k_{w} z+\theta\right) .
\end{gathered}
$$

We first determine the resonant frequencies by obtaining a first-order straightforward perturbation expansion in Sec. II.

\section{A STRAIGHTFORWARD EXPANSION}

We seek an asymptotic expansion for $\psi$ of the form

$$
\psi(x, z)=\psi_{0}(x, z)+\epsilon \psi_{1}(x, z)+\cdots \text {. }
$$

Substituting Eq. (8) into Eqs. (5) - (7), transferring the boundary conditions to $x=0$ and $x=1$ by developing $\psi$ and its derivatives in Taylor series around $x=0$ and $x=1$, and equating coefficients of equal powers of $\epsilon$, we obtain

$O\left(\epsilon^{0}\right)$

$$
\begin{array}{ll}
\nabla^{2} \psi_{0}+k^{2} \psi_{0}=0, \\
\psi_{0}=0 \quad \text { at } x=0, \\
\psi_{0}=0 \quad \text { at } x=1,
\end{array}
$$

$\mathbf{O}(\epsilon)$

$$
\nabla^{2} \psi_{1}+k^{2} \psi_{1}=0
$$

$$
\begin{aligned}
\left(\frac{\partial^{2}}{\partial z^{2}}+k^{2}\right) \psi_{1}= & -\sin \left(k_{w} z\right)\left(\frac{\partial^{2}}{\partial z^{2}}+k^{2}\right) \frac{\partial \psi_{0}}{\partial x} \\
& -k_{w} \cos \left(k_{w} z\right) \frac{\partial^{2} \psi_{0}}{\partial x \partial z} \quad \text { at } x=0,
\end{aligned}
$$




$$
\begin{aligned}
\left(\frac{\partial^{2}}{\partial z^{2}}+k^{2}\right) \psi_{1}= & -\sin \left(k_{w} z+\theta\right)\left(\frac{\partial^{2}}{\partial z^{2}}+k^{2}\right) \frac{\partial \psi_{0}}{\partial x} \\
& -k_{w} \cos \left(k_{w} z+\theta\right) \frac{\partial^{2} \psi_{0}}{\partial x \partial z} \text { at } x=1 .
\end{aligned}
$$

The solution of Eq. (9) that satisfies the boundary conditions (10) and (11) is

$$
\psi_{0}=A_{n} \sin (n \pi x) \exp \left(i k_{n} z\right),
$$

where $n=1,2,3, \ldots$, and for a propagating mode

$$
k_{n}^{2}=k^{2}-(n \pi)^{2}>0 \text {. }
$$

Substituting Eq. (15) into the boundary conditions (13) and (14), we obtain

$$
\begin{aligned}
&\left(\frac{\partial^{2}}{\partial z^{2}}+k^{2}\right) \psi_{1}= \frac{1}{2} i n \pi A_{n}\left\{\left(n^{2} \pi^{2}-k_{n} k_{w}\right) \exp \left[i\left(k_{n}+k_{w}\right) z\right]-\left(n^{2} \pi^{2}\right.\right. \\
&\left.\left.+k_{n} k_{w}\right) \exp \left[i\left(k_{n}-k_{w}\right) z\right]\right\} \text { at } x=0, \\
&\left(\frac{\partial^{2}}{\partial z^{2}}+k^{2}\right) \psi_{1}= \frac{1}{2} i n \pi(-1)^{n} A_{n}\left\{( n ^ { 2 } \pi ^ { 2 } - k _ { n } k _ { w } ) \operatorname { e x p } \left[i\left(k_{n}+k_{w}\right) z\right.\right. \\
&\left.+i \theta]-\left(n^{2} \pi^{2}+k_{n} k_{w}\right) \exp \left[i\left(k_{n}-k_{w}\right) z-i \theta\right]\right\} \\
& \text { at } x=1
\end{aligned}
$$

Equations (17) and (18) suggest that $\psi_{1}$ has a particular solution of the form

$$
\begin{aligned}
\psi_{1}(x, z)= & \frac{1}{2} i n \pi A_{n}\left\{\left(n^{2} \pi^{2}-k_{n} k_{w}\right) \Phi_{1}(x) \exp \left[i\left(k_{n}+k_{w}\right) z\right]\right. \\
& \left.-\left(n^{2} \pi^{2}+k_{n} k_{w}\right) \Phi_{2}(x) \exp \left[i\left(k_{n}-k_{w}\right) z\right]\right\} .
\end{aligned}
$$

Substituting Eq. (19) into Eqs. (12)-(14), we obtain

$$
\begin{aligned}
& \frac{d^{2} \Phi_{1}}{d x^{2}}+\alpha_{1}^{2} \Phi_{1}=0, \quad \alpha_{1}^{2}=k^{2}-\left(k_{n}+k_{w}\right)^{2}, \\
& \Phi_{1}(0)=\alpha_{1}^{-2}, \\
& \Phi_{1}(1)=(-1)^{n} \alpha_{1}^{-2} \exp (i \theta), \\
& \frac{d^{2} \Phi_{2}}{d x^{2}}+\alpha_{2}^{2} \Phi_{2}=0, \quad \alpha_{2}^{2}=k^{2}-\left(k_{n}-k_{w}\right)^{2}, \\
& \Phi_{2}(0)=\alpha_{2}^{-2}, \\
& \Phi_{2}(1)=(-1)^{n} \alpha_{2}^{-2} \exp (-i \theta) .
\end{aligned}
$$

The solutions of Eqs. (20)-(25) are

$\Phi_{j}=\left[\alpha_{j}^{2} \sin \left(\alpha_{j}\right)\right]^{-1}\left(\left[(-1)^{n} \exp [-i \theta \cos (\pi j)]-\cos \left(\alpha_{j}\right)\right.\right.$

$$
\left.x \sin \left(\alpha_{j} x\right)+\sin \left(\alpha_{j}\right) \cos \left(\alpha_{j} x\right)\right),
$$

where $j=1,2$.

We note that $\Phi_{j} \rightarrow \infty$ as $\alpha_{j} \rightarrow m \pi$ for integer $m$. In other words, the straightforward expansion breaks down near the resonant wave numbers

$$
k_{r}^{2}=\left(k_{n} \pm k_{w}\right)^{2}+m^{2} \pi^{2} .
$$

Uniformly valid expansions in the neighborhood of resonance will be obtained in Sec. III by using the method of multiple scales. ${ }^{3}$ Since $k_{r}^{2}-m^{2} \pi^{2}=k_{m}^{2}$, the resonance condition can be written

$$
k_{m}=k_{n} \pm k_{w} \text {. }
$$

\section{EXPANSIONS VALID NEAR RESONANCE}

We seek an asymptotic expansion of the form

$$
\psi(x, z)=\psi_{0}\left(x, z_{0}, z_{1}\right)+\epsilon \psi_{1}\left(x, z_{0}, z_{1}\right)+\cdots,
$$

where $z_{0}=z$ is a length scale of the order of a wavelength and $z_{1}=\epsilon z$ is a long length scale characterizing the amplitude and phase modulations. Substituting Eq. (29) into Eqs. (5) $-(7)$ and equating coefficients of equal powers of $\epsilon$, we obtain

$\mathbf{O}\left(\epsilon^{0}\right)$

$$
\begin{aligned}
& \frac{\partial^{2} \psi_{0}}{\partial x^{2}}+\frac{\partial^{2} \psi_{0}}{\partial z_{0}^{2}}+k^{2} \psi_{0}=0, \\
& \psi_{0}=0 \quad \text { at } x=0, \\
& \psi_{0}=0 \quad \text { at } x=1,
\end{aligned}
$$

$O(\epsilon)$

$$
\begin{aligned}
\frac{\partial^{2} \psi_{1}}{\partial x^{2}}+\frac{\partial^{2} \psi_{1}}{\partial z_{0}^{2}}+k^{2} \psi_{1} & =-2 \frac{\partial^{2} \psi_{0}}{\partial z_{0} \partial z_{1}} \\
\left(\frac{\partial^{2}}{\partial z_{0}^{2}}+k^{2}\right) \psi_{1}= & -\sin \left(k_{w} z_{0}\right)\left(\frac{\partial^{2}}{\partial z_{0}^{2}}+k^{2}\right) \frac{\partial \psi_{0}}{\partial x} \\
& -k_{w} \cos \left(k_{w} z_{0}\right) \frac{\partial^{2} \psi_{0}}{\partial x \partial z_{0}}-2 \frac{\partial^{2} \psi_{0}}{\partial z_{0} \partial z_{1}} \quad \text { at } x=0 \\
\left(\frac{\partial^{2}}{\partial z_{0}^{2}}+k^{2}\right) \psi_{1}= & -\sin \left(k_{w} z_{0}+\theta\right)\left(\frac{\partial^{2}}{\partial z_{0}^{2}}+k^{2}\right) \frac{\partial \psi_{0}}{\partial x} \\
& -k_{w} \cos \left(k_{w} z_{0}+\theta\right) \frac{\partial^{2} \psi_{0}}{\partial x \partial z_{0}}-2 \frac{\partial^{2} \psi_{0}}{\partial z_{0} \partial z_{1}} \text { at } x=1
\end{aligned}
$$

The solution of Eq. (30) for the case of resonance must contain both of the interacting modes, i.e., $\psi_{0}=A_{n}\left(z_{1}\right) \sin (n \pi x) \exp \left(i k_{n} z_{0}\right)+A_{m}\left(z_{1}\right) \sin (m \pi x) \exp \left(i k_{m} z_{0}\right)$

where $A_{n}\left(z_{1}\right)$ and $A_{m}\left(z_{1}\right)$ are to be determined at the next level of approximation. Substituting for $\psi_{0}$ into Eqs. (33) - (35), we obtain

$$
\begin{aligned}
\frac{\partial^{2} \psi_{1}}{\partial x^{2}}+\frac{\partial^{2} \psi_{1}}{\partial z_{0}^{2}}+k^{2} \psi_{1}= & -2 i k_{n} A_{n}^{\prime} \sin (n \pi x) \exp \left(i k_{n} z_{0}\right) \\
& -2 i k_{m} A_{m}^{\prime} \sin (m \pi x) \exp \left(i k_{m} z_{0}\right) \\
\left(\frac{\partial^{2}}{\partial z_{0}^{2}}+k^{2}\right) \psi_{1}= & \frac{1}{2} i \pi\left(\sum_{j=n, m} j\left(j^{2} \pi^{2}-k_{j} k_{w}\right) A_{j} \exp \left[i\left(k_{j}+k_{w}\right) z_{0}\right]\right. \\
- & \left.\sum_{j=n, m} j\left(j^{2} \pi^{2}+k_{j} k_{w}\right) A_{j} \exp \left[i\left(k_{j}-k_{w}\right) z_{0}\right]\right)
\end{aligned}
$$

$$
\begin{aligned}
\left(\frac{\partial^{2}}{\partial z_{0}^{2}}+k^{2}\right) \psi_{1}= & \frac{1}{2} i \pi\left(\sum _ { j = n , m } ( - 1 ) ^ { j } j ( j ^ { 2 } \pi ^ { 2 } - k _ { j } k _ { w } ) A _ { j } \operatorname { e x p } \left[i \left(k_{j}\right.\right.\right. \\
& \left.\left.+k_{w}\right) z_{0}+i \theta\right]-\sum_{j=n, m}(-1)^{j} j\left(j^{2} \pi^{2}+k_{j} k_{w}\right) A_{j} \\
& \left.\times \exp \left[i\left(k_{j}-k_{w}\right) z_{0}-i \theta\right]\right) \quad \text { at } x=1,
\end{aligned}
$$

where primes denote differentiation with respect to $z_{1}$. Since the homogeneous equations $(37)-(39)$ have a nontrivial solution, the inhomogeneous equations (37)-(39) have a solution if, and only if, a solvability condition is satisfied. To determine this solvability condition, we make use of the resonance condition given by Eq. (28). We note that Eq. (28) indicates that two resonant cases with different values of $m$ are possible. The terms on the right-hand side of Eqs. (38) and (39) that lead to resonance are the same for both values of $m$ except that when $k_{m}=k_{n}+k_{w w}$, the roles of $k_{m}$ and $k_{n}$ are interchanged. Thus resonance occurs whenever $k_{w}$ is nearly 
equal to the difference between the wave numbers of two propagating modes. We consider the case of near resonance $k_{m} \approx k_{n}-k_{w}$ and introduce a detuning parameter $\delta$ defined by

$$
k_{m}=k_{n}-k_{w}+\epsilon \delta, \quad \delta=\mathrm{O}(1),
$$

and express $\exp \left[i\left(k_{n}-k_{w}\right) z_{0}\right]$ and $\exp \left[i\left(k_{m}+k_{w}\right) z_{0}\right]$ as

$$
\begin{aligned}
& \exp \left[i\left(k_{n}-k_{w}\right) z_{0}\right]=\exp \left[i k_{m} z_{0}-i \delta z_{1}\right] \\
& \exp \left[i\left(k_{m}+k_{w}\right) z_{0}\right]=\exp \left[i k_{n} z_{0}+i \delta z_{1}\right]
\end{aligned}
$$

To determine the solvability condition for Eqs. (37)-(39) we seek a particular solution of the form

$$
\psi_{1}=i \Phi_{n}\left(x, z_{1}\right) \exp \left(i k_{n} z_{0}\right)+i \Phi_{m}\left(x, z_{1}\right) \exp \left(i k_{m} z_{0}\right) \text { 。 }
$$

Substituting Eqs. (42) into Eqs. (37)-(39), using Eq. (41), and equating the coefficients of $\exp \left(i k_{n} z_{0}\right)$ and $\exp \left(i k_{m} z_{0}\right)$ on both sides, we obtain

$$
\begin{aligned}
& \frac{\partial^{2} \Phi_{n}}{\partial x^{2}}+n^{2} \pi^{2} \Phi_{n}=-2 k_{n} A_{n}^{\prime} \sin (n \pi x), \\
& n^{2} \pi^{2} \Phi_{n}=\frac{1}{2} m \pi A_{m}\left(m^{2} \pi^{2}-k_{m} k_{w}\right) \exp \left(i \delta z_{1}\right) \text { at } x=0 \text {, } \\
& n^{2} \pi^{2} \Phi_{n}=\frac{1}{2} m \pi(-1)^{m} A_{m}\left(m^{2} \pi^{2}-k_{m} k_{w}\right) \exp \left[i\left(\delta z_{1}+\theta\right)\right] \\
& \text { at } x=1 \text {, } \\
& \frac{\partial^{2} \Phi_{m}}{\partial x^{2}}+m^{2} \pi^{2} \Phi_{m}=-2 k_{m} A_{m}^{\prime} \sin (m \pi x), \\
& m^{2} \pi^{2} \Phi_{m}=-\frac{1}{2} n \pi\left(n^{2} \pi^{2}+k_{n} k_{w}\right) A_{n} \exp \left(-i \delta z_{1}\right) \\
& \text { at } x=0 \text {, } \\
& m^{2} \pi^{2} \Phi_{m}=\frac{1}{2} n \pi(-1)^{n+1}\left(n^{2} \pi^{2}+k_{n} k_{w}\right) A_{n} \exp \left[-i\left(\delta z_{1}+\theta\right)\right] \\
& \text { at } x=1 \text {. }
\end{aligned}
$$

The general solution of $\mathrm{Eq}$. (43a) is

$\Phi_{n}\left(x, z_{1}\right)=c_{1} \cos (n \pi x)+c_{2} \sin (n \pi x)+\left(k_{n} / n \pi\right) x A_{n}^{\prime} \cos (n \pi x)$.

Substituting Eq. (45) into Eqs. (43b) and (43c) yields

$$
\begin{aligned}
n^{2} \pi^{2} c_{1}=\frac{1}{2} m \pi A_{m}\left(m^{2} \pi^{2}-k_{m} k_{w}\right) & \exp \left(i \delta z_{1}\right), \\
(-1)^{n} n^{2} \pi^{2}\left[c_{1}+\left(k_{n} / n \pi\right) A_{n}^{\prime}\right]= & \frac{1}{2} m \pi(-1)^{m} A_{m}\left(m^{2} \pi^{2}-k_{m} k_{w}\right) \\
& \times \exp \left[i\left(\delta z_{1}+\theta\right)\right] .
\end{aligned}
$$

Eliminating $c_{1}$ from Eqs. (46a) and $46 \mathrm{~b}$ ), we obtain the following solvability condition for Eqs. (43a)-(43c):

$A_{n}^{\prime}=\frac{1}{2}\left(m / n k_{n}\right) A_{m}\left(k_{m} k_{w}-m^{2} \pi^{2}\right)\left[1-(-1)^{m+m} \exp (i \theta)\right] \exp \left(i \delta z_{1}\right)$.

Similarly, the solvability condition for Eqs. (44a)-(44c) is

$$
\begin{aligned}
A_{m}^{\prime}= & \frac{1}{2}\left(n / m k_{m}\right) A_{n}\left(n^{2} \pi^{2}+k_{n} k_{w}\right)\left[1-(-1)^{m+n} \exp (-i \theta)\right] \\
& \times \exp \left(-i \delta z_{1}\right) .
\end{aligned}
$$

We seek a solution to Eqs. (47) and (48) of the form

$$
A_{m}=a_{m} \exp \left(s z_{1}\right), \quad A_{n}=a_{n} \exp \left[(s+i \delta) z_{1}\right],
$$

where $a_{j}$ and $s$ are constants. Substituting Eq. (49) into Eqs. (47) and (48) and eliminating the $a^{\prime} s$, we obtain

$$
s(s+i \delta)=\Omega \text {, }
$$

where

$\Omega=\frac{1}{2}\left(k_{m} k_{n}\right)^{-1}\left(k_{m} k_{w}-m^{2} \pi^{2}\right)\left(k_{n} k_{w}+n^{2} \pi^{2}\right)\left[1-(-1)^{m+n} \cos \theta\right]$

The solution of $\mathrm{Eq} .(50 \mathrm{a})$ is

$$
s_{1,2}=\frac{1}{2} i\left[-\delta \pm\left(\delta^{2}-4 \Omega\right)^{1 / 2}\right] \text {. }
$$

The coupling coefficient $\Omega$ is negative because

$$
k_{m} k_{w}-m^{2} \pi^{2}=k_{n} k_{m}-k^{2}+\epsilon \delta k_{m}<0,
$$

since $k_{n}<k$ and $k_{m}<k$. Hence, $s$ is purely imaginary and $A_{m}$ and $A_{n}$ are consequently bounded.

From Eqs. (49) and (50) we note that for two modes to interact the coupling coefficient $\Omega$ must be different from zero. For the special case of a guide of periodically varying direction $(\theta=0), m+n$ must be an odd integer; $i_{.}$e., odd modes generate even modes, or vice versa. For the case of a guide with periodically varying thickness $(\theta=\pi), m+n$ must be an even integer; i.e., odd (even) modes generate odd (even) modes. For values of $\theta$ different from 0 and $\pi, m$ takes on all integer values.

The exact forms of $A_{n}\left(z_{1}\right)$ and $A_{m}\left(z_{1}\right)$ depend on the excitation. Let the excitation at $z=0$ be

$$
A_{n}(0)=1, \quad A_{m}(0)=0 .
$$

According to Eq. (49), $A_{n}\left(z_{1}\right)$ and $A_{m}\left(z_{1}\right)$ are of the form

$$
\begin{aligned}
& A_{n}=a_{1} \exp \left[\left(s_{1}+i \delta\right) z_{1}\right]+a_{2} \exp \left[\left(s_{2}+i \delta\right) z_{1}\right], \\
& A_{m}=a_{3} \exp \left(s_{1} z_{1}\right)+a_{4} \exp \left(s_{2} z_{1}\right),
\end{aligned}
$$

where the $a_{i}$ are complex constants.We substitute Eqs. (53a) and (53b) into Eqs. (52), (47), and (48), evaluate the latter at $z_{1}=0$, and obtain

$$
\begin{aligned}
& a_{1}+a_{2}=1, \\
& a_{3}+a_{4}=0, \\
& \left(s_{1}+i \delta\right) a_{1}+\left(s_{2}+i \delta\right) a_{2}=0, \\
& s_{1} a_{3}+s_{2} a_{4}=\gamma\left(n / m k_{m}\right)\left(n^{2} \pi^{2}+k_{n} k_{w}\right),
\end{aligned}
$$

where $\gamma=\frac{1}{2}\left[1-(-1)^{m+n} \exp (-i \theta)\right]$.

Solving for the $a^{\prime} s$ and substituting the result into Eqs. (53a) and (53b), we obtain

$$
\begin{aligned}
A_{n}= & \left(s_{1}-s_{2}\right)^{-1}\left\{-\left(s_{2}+i \delta\right) \exp \left[\left(s_{1}+i \delta\right) z_{1}\right]\right. \\
& \left.+\left(s_{1}+i \delta\right) \exp \left[\left(s_{2}+i \delta\right) z_{1}\right]\right\}, \\
A_{m}= & \left(s_{1}-s_{2}\right)^{-1} \gamma\left(n / m k_{m}\right)\left(n^{2} \pi^{2}+k_{n} k_{w}\right)\left[\exp \left(s_{1} z_{1}\right)\right. \\
& \left.-\exp \left(s_{2} z_{1}\right)\right] .
\end{aligned}
$$

We can express $A_{n}$ and $A_{m}$ in the form

$$
A_{j}=\left|A_{j}\right| \exp \left(i B_{j}\right) \text {, }
$$

where

$$
\left|A_{j}\right|=\left[\left(\operatorname{Re} A_{\jmath}\right)^{2}+\left(\operatorname{Im} A_{\jmath}\right)^{2}\right]^{1 / 2},
$$

and

$$
\beta_{j}=\tan ^{-1}\left(\operatorname{Im} A_{j} / \operatorname{Re} A_{j}\right) .
$$

Substituting for $s_{1}$ and $s_{2}$ from Eq. (51) into Eqs. (55a) and $(55 \mathrm{~b})$, we obtain

$$
\left|A_{n}\right|=\left(\delta^{2}-4 \Omega\right)^{-1 / 2}\left\{\delta^{2}-4 \Omega \cos ^{2}\left[\frac{1}{2}\left(\delta^{2}-4 \Omega\right)^{1 / 2} z_{1}\right]\right\}^{1 / 2},
$$




$$
\begin{aligned}
\left|A_{m}\right|= & \left(n / m k_{m}\right)\left(n^{2} \pi^{2}+k_{n} k_{w o}\right) \\
& \times\left(\frac{1-(-1)^{m+n} \cos \theta}{\delta^{2}-4 \Omega}\right)^{1 / 2} \sin \left[\frac{1}{2}\left(\delta^{2}-4 \Omega\right)^{1 / 2} z_{1}\right] .
\end{aligned}
$$

If these expressions are now substituted into Eq. (36) and then the Poynting vector is evaluated and integrated over the cross section of the guide, one can show that energy is conserved. In other words, the energy of the excitation at $z=0$ is equal to the sum of the energies of the two interacting modes at any location along the $z$ axis.

\section{CONCLUSIONS}

The analysis shows how sinusoidal wall perturbations result in the generation of a propagating mode from an excited mode. The energy is exchanged between the two modes in a manner consistent with conservation of energy. This feature of the problem can be utilized in the design of mode couplers where it is desired to transfer energy from one propagating mode into another whenever $k_{m} \approx k_{n}-k_{w}$.

${ }^{1}$ D. Marcuse, Light Transmission Optics (Van Nostrand, New York, 1972)

${ }^{2}$ A. H. Hayfeh, J. A coust. Soc. Am. (to be published).

${ }^{3}$ A. H. Nayfeh, Perturbation Methods (Interscience, New York, 1973). 